\title{
openheart Music intervention to relieve anxiety and pain in adults undergoing cardiac surgery: a systematic review and meta- analysis
}

\author{
Ellaha Kakar (D) , ${ }^{1,2}$ Ryan J Billar, ${ }^{3}$ Joost van Rosmalen (D) , ${ }^{4}$ Markus Klimek, ${ }^{5}$ \\ Johanna J M Takkenberg, ${ }^{6}$ Johannes Jeekel ${ }^{1,2}$
}

To cite: Kakar E, Billar RJ, van Rosmalen J, et al. Music intervention to relieve anxiety and pain in adults undergoing cardiac surgery: a systematic review and meta-analysis. Open Heart 2021;7:e001474.

doi:10.1136/

openhrt-2020-001474

EK and RJB are joint first authors.

Received 5 October 2020 Revised 26 November 2020 Accepted 1 December 2020

Check for updates

(C) Author(s) (or their employer(s)) 2021. Re-use permitted under CC BY-NC. No commercial re-use. See rights and permissions. Published by BMJ.

${ }^{1}$ Surgery, Erasmus MC, Rotterdam, South-Holland, Netherlands

${ }^{2}$ Neuroscience, Erasmus MC, Rotterdam, South-Holland, The Netherlands

${ }^{3}$ Pediatric Surgery, Erasmus MC, Rotterdam, South-Holland, Netherlands

${ }^{4}$ Biostatics, Erasmus MC, Rotterdam, South-Holland, Netherlands

${ }^{5}$ Anesthesiology, Erasmus MC, Rotterdam, South-Holland, Netherlands

${ }^{6}$ CardioThoracic Surgery, Erasmus University Medical Center, Rotterdam, SouthHolland, Netherlands

Correspondence to Ms Ellaha Kakar; e.kakar@ erasmusmc.nl

\section{ABSTRACT}

Objectives Previous studies have reported beneficial effects of perioperative music on patients' anxiety and pain. We performed a systematic review and metaanalysis of randomised controlled trials investigating music interventions in cardiac surgery.

Methods Five electronic databases were systematically searched. Primary outcomes were patients' postoperative anxiety and pain. Secondary outcomes were hospital length of stay, opioid use, vital parameters and time on mechanical ventilation. PRISMA guidelines were followed and PROSPERO database registration was completed (CRD42020149733). A meta-analysis was performed using random effects models and pooled standardised mean differences (SMD) with 95\% confidence intervals were calculated.

Results Twenty studies were included for qualitative analysis (1169 patients) and 16 (987 patients) for metaanalysis. The first postoperative music session was associated with significantly reduced postoperative anxiety $(\mathrm{SMD}=-0.50(95 \% \mathrm{Cl}-0.67$ to -0.32$), \mathrm{p}<0.01)$ and pain $(\mathrm{SMD}=-0.51(95 \% \mathrm{Cl}-0.84$ to -0.19$), \mathrm{p}<0.01)$. This is equal to a reduction of 4.00 points $(95 \% \mathrm{Cl} 2.56$ to 5.36$)$ and 1.05 points $(95 \% \mathrm{Cl} 0.67$ to 1.41$)$ on the State-Trait Anxiety Inventory and Visual Analogue Scale (VAS)/Numeric Rating Scale (NRS), respectively, for anxiety, and 1.26 points (95\% Cl 0.47 to 2.07 ) on the VAS/NRS for pain. Multiple days of music intervention reduced anxiety until 8 days postoperatively $(\mathrm{SMD}=-0.39(95 \% \mathrm{Cl}-0.64$ to $-0.15), p<0.01$ ).

Conclusions Offering recorded music is associated with a significant reduction in postoperative anxiety and pain in cardiac surgery. Unlike pharmacological interventions, music is without side effects so is promising in this population.

\section{INTRODUCTION}

Patients undergoing cardiac surgery often have perioperative anxiety and severe postoperative pain, despite the administration of benzodiazepines and opioids. ${ }^{1-4}$ Postoperative admission to the intensive care unit (ICU) exposes them to stressors known to increase anxiety and pain, such as noise,

\section{Key questions}

What is already known about this subject?

- Perioperative anxiety and pain are prevalent in patients undergoing cardiac surgery, despite the use of pharmacological interventions with well-known side effects.

- Music intervention has provided promising results in surgical patients.

What does this study add?

- The results of this study provide some evidence for the beneficial effect of perioperative music intervention on postoperative anxiety and pain in patients undergoing cardiac surgery.

How might this impact on clinical practice?

- Since music intervention has neither risks nor known side effects but may have a positive effect on the patients' health outcomes, healthcare professionals should consider providing perioperative music for patients undergoing cardiac surgery.

sleeplessness, mechanical ventilation and immobility. These stressors may lead to longer hospitalisation and higher use of benzodiazepines and opioids, with their inherent risk of side effects and adverse events. ${ }^{5-14}$ Research efforts have been directed towards approaches to relieve anxiety and pain. Apart from pharmacological therapies, nonpharmacological therapies have provided promising results. ${ }^{15}$

A music intervention is relatively inexpensive and an easily applicable nonpharmaceutical intervention which has no known side effects. Previous studies in mixed surgical populations have found statistically significant beneficial effects of perioperative recorded music on patients' anxiety, pain and neurohormonal stress response, with less consumption of intraoperative sedatives and postoperative opioids. ${ }^{15-19}$ However, these 
effects may not directly apply to patients undergoing highly invasive cardiac surgical procedures.

We performed a systematic review and meta-analysis to assess and quantify the effect of perioperative recorded music interventions on anxiety and pain in adult patients undergoing cardiac surgery. To the best of our knowledge, this is the first study of this kind.

\section{METHODS}

This systematic review and meta-analysis was performed according to the Preferred Reporting Items for Systematic Reviews and Meta-analysis (PRISMA) guidelines (see online supplementary data S1) and was registered in the PROSPERO database (https://www.crd.york.ac.uk/ PROSPERO) as record number CRD42020149733. ${ }^{20}$

\section{Search strategy}

With the help of a dedicated biomedical information specialist, we performed a search in the Embase, Medline Ovid, Web of Science, Cochrane CENTRAL and Google scholar databases for studies between 1 January 1992 and 25 October 2019. Keywords used in the literature search were "music" and "cardiac surgery". Online Supplementary data S2 gives a detailed description of the search terms and results per database. Reference lists of included studies were manually screened for additional studies that met the inclusion criteria.

\section{Study screening and selection}

Two authors (EK/RJB) independently screened all identified articles on title/abstract and full text according to a standardised protocol. ${ }^{21}$ We have included studies with $(\mathrm{P})$ adult patients undergoing cardiac surgery investigating the effect of (I) perioperative recorded music compared with a (C) control group on $(\mathrm{O})$ anxiety and pain in (S) randomised controlled trials (RCTs). PICOS is a mnemonic used in evidence based medicine and stands for, respectively; Patient, Intervention, Control, Outcomes, and Study ${ }^{22}$ type. Other inclusion criteria were assessment of surrogates of anxiety and pain (use of opioids and vital parameters), full text available in the English language and the use of perioperative recorded music in a hospital. Studies investigating the effect of music during a postoperative painful procedure (eg, chest tube removal, positioning of the patient) were excluded since such procedures reflect only a fraction of postoperative anxiety and pain during hospitalisation. Music interventions were defined as the use of recorded music consisting of melody, harmony and rhythm, and offered in a hospital setting. Nature sounds were considered only when used in addition to music. If studies compared a music group to multiple other groups, the group without music that was most similar to the music group with respect to the intervention was chosen as the control group (eg, if groups were 'scheduled rest' and 'standard care', 'scheduled rest' was chosen as the control group if the music group also received a resting period).
Any disagreements between reviewers were resolved by the senior author $(\mathrm{JJ})$.

\section{Data collection process and data items}

Two of the authors (EK/RJB) independently extracted and checked the data from eligible studies according to a pre-specified dataset. The following study characteristics were extracted: author name, year of publication, sex, mean age, type of cardiac surgery, surgical method and numbers of patients in the intervention and control groups. The following intervention characteristics were extracted: type of music (genre, rhythm, beats per minute, how music was defined (eg, 'soothing music'), timing of the intervention (before, during or after surgery) and duration of the intervention (in minutes). Primary outcomes were mean anxiety and pain scores assigned on the guidance of validated subjective assessment tools at baseline and after the intervention. Secondary outcomes were hospital length of stay (LOS, days), opioid use (morphine equivalents; $\mathrm{mg}$ ), systolic blood pressure (SBP, $\mathrm{mmHg}$ ), diastolic blood pressure (DBP, $\mathrm{mmHg}$ ), mean arterial pressure (MAP, $\mathrm{mmHg}$ ), heart rate (HR, beats/min), respiratory rate (RR, breaths/min), oxygen saturation $(\mathrm{SpO} 2, \%)$, and time on mechanical ventilation (min).

\section{Quantitative analysis}

Statistical analysis

Descriptive statistics are presented as means (SD), counts and percentages. For the main analysis, a meta-analysis was performed for all primary and secondary outcomes (except opioid use) using data for the first postoperative music session with immediate assessment of anxiety and/or pain. When multiple music sessions were applied, only data for the first postoperative session were used. The meta-analysis was performed using random effects models to calculate the overall treatment effect based on standardised mean differences (SMD), accounting for between-study heterogeneity. The between-study variance was calculated with the restricted maximum likelihood method. The level of heterogeneity was assessed using the $\mathrm{I}^{2}$ statistic. Studies were included for quantitative analysis when mean values and corresponding SDs of the main and secondary outcomes were reported. Studies with significant differences in anxiety and/or pain scores at baseline were excluded from the quantitative analysis. To calculate the reduction for pain and for anxiety based on the pooled SMD of the meta-analysis, a back-transformation was applied to the anxiety and pain scores as described by the Cochrane Handbook for Systematic Reviews. ${ }^{23}$ For this back-transformation, the SDs of anxiety and pain were estimated by pooling the SDs of the control groups for the different assessment tools separately.

In an additional analysis, meta-analyses were performed for the primary outcomes using the data for the last session when multiple sessions were applied. For the primary outcomes, subgroup meta-analysis was 
performed on timing of the intervention (preoperatively, intraoperatively or postoperatively), how the music choice was assessed (eg, by the researcher or preference of the patient) and risk of bias due to the randomisation procedure (low risk was defined when both random sequence generation and allocation concealment were scored as low risk, other studies were scored as high risk of bias). For use of opioids, a random effects metaanalysis was performed to assess the effect of the intervention on postoperative opioid use; where necessary, opioid use was accumulated over multiple postoperative days. If multiple types of opioids were administered, daily dosages were converted to morphine equivalents and summed to calculate the total daily opioid use. The total dosage of opioids during the postoperative assessment period was determined by pooling the total daily dosages using formulas for pooled variance. Data were analysed using $\mathrm{R}$ version 3.6.3 and a two-sided $\mathrm{p}$ value $<0.05$ was considered statistically significant.

\section{Qualitative analysis}

\section{Risk of bias assessment}

Selected articles were independently evaluated on risk of bias by the same two authors according to the Cochrane Risk of Bias tool for RCTs. ${ }^{23}$ Incomplete data outcome due to drop-out of $\geq 10 \%$ was considered as high risk of bias. If the study protocol was not available, the risk for selective reporting was considered unclear. Funnel plots were made to assess publication bias. The GRADE (Grading of Recommendations, Assessment, Development and Evaluations) criteria were used to assess the quality of the effect of music on anxiety and pain, and to make clinical practice recommendations. ${ }^{23} 24$

\section{RESULTS}

The literature search yielded 1537 results. After removal of duplicates, the titles and abstracts of 1018 articles were screened for relevance. Eventually, the full text of 36 of these articles and two additional articles were assessed for inclusion in the review. On the basis of the inclusion and exclusion criteria, 20 studies were included for the qualitative analysis (1169 patients) and 16 of these 20 studies (987 patients) for meta-analysis (see figure 1$).{ }^{25-44}$ The rate of agreement between the two reviewers was considered high $(80 \%)$ on study selection and inclusion, risk of bias assessment and data extraction.

\section{Study characteristics}

A detailed description of the study and music intervention characteristics is shown in online supplemental data S3. Cardiothoracic surgical procedures were mostly coronary artery bypass graft and/or valve replacement (90\%). None of the papers described the surgical method used. Eleven studies assessed anxiety (State Trait Anxiety Inventory (STAI, 4/11), Visual Analogue Scale (VAS, 4/11) and Numeric Rating Scale (NRS, 1/11), Hospital Anxiety and Depression Scale (HADS, 1/11), STAI and NRS (1/11)) and 10 assessed pain (VAS (55.6\%), NRS (33.3\%), Visual Rating Scale (VRS, 11.1\%)).

The type of music offered was commonly described as relaxing, calming, soft, sedative, light and absence of strong rhythms and percussion $(60 \%)$. It was mostly offered through headphones $(70 \%)$. The played music selection was either chosen by the patient from preselected music lists $(40 \%)$, selected by the researcher $(35 \%)$ or self-chosen by the patient $(15 \%)$. Two studies used a combination of the above-described methods of music selection (10\%). ${ }^{31} 43$ Twelve studies (60\%) provided multiple music sessions, either repeated on a day or days or once daily over several day(s). Patients in the control groups received a scheduled rest (eight studies, $40 \%$ ), standard care (six studies, 30\%), headphones/earphones without music (four studies, 20\%), breathing exercises (one study, 10\%) or an intraoperative blank tape combined with postoperative standard care (one study, 10\%).

Fourteen studies solely provided postoperative music, of which seven were in the ICU, ${ }^{27} 323435384243$ two in the surgical ward, ${ }^{25} 39$ one in the ICU and ward ${ }^{26}$ and four did not specify. ${ }^{28} 29{ }^{44}$ Five studies provided postoperative music in addition to preoperative and/or intraoperative music. $^{31} 33364041$ One study provided both preoperative and intraoperative music. ${ }^{30}$ In this study, reported outcome parameters during hospitalisation were assessed until postoperative day 8 and the duration of each music intervention was at least $20 \mathrm{~min}$.

\section{Risk of bias assessment}

Figure 2 presents a detailed overview of the risk of bias assessment. Overall, the risk of bias was found to be moderate to high. A high risk of selection bias was considered present in three studies (15\%) due to open random allocation list, ${ }^{27}$ random sequence generation based on odd and even numbers ${ }^{33}$ and availability of the intervention, ${ }^{43}$ respectively. Five studies provided insufficient details regarding the random sequence generation $(20 \%)^{34} 39404244$ and 11 studies (55\%) regarding the allocation concealment. ${ }^{25} 28313437-4244$ An overall high risk of performance bias was present, as blinding of patients to the intervention is only feasible when the intervention is administered during general anaesthesia. Three studies (15\%) reported blinding of the personnel. ${ }^{38} 4041$ Two studies $(10 \%)$ reported a high risk of reporting bias, as outcome parameters in the research protocols differed from those in the published articles. ${ }^{33} 35$ Other risk of bias was considered high in two studies $(10 \%)$; in one of these the patients in the control group were significantly older than the patients in the music group ${ }^{40}$ and the other lacked information on baseline characteristics such as age, sex and type of surgery. ${ }^{44}$ A summary of the risk of bias is shown in figure 3 . The funnel plots investigating bias of studies on the effect of perioperative music on anxiety and pain showed a near funnel-shaped plot (online supplemental data S4 and S5). 


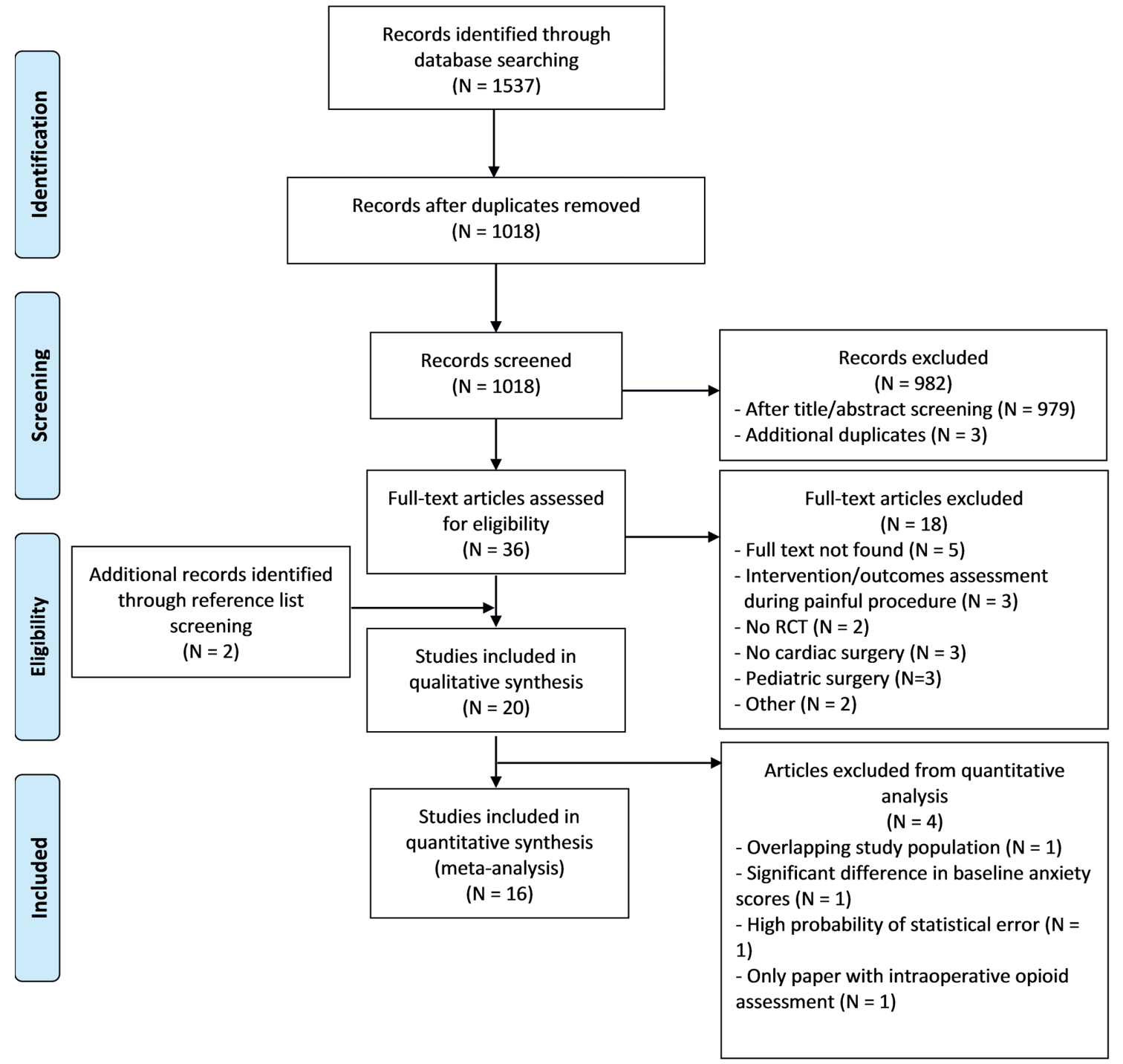

Figure 1 PRISMA flow diagram. $\mathrm{N}$ indicates the number of articles.

\section{Effect of music intervention on postoperative anxiety}

Anxiety scores were pooled after the first postoperative music session in nine of the 11 studies. ${ }^{25-27} 30-333644$ The scores of two of these 11 studies could not be pooled because SDs were lacking. ${ }^{28} 29$ The pooled analysis resulted in a significant effect of perioperatively offered music on postoperative anxiety $(\mathrm{SMD}=-0.50(95 \% \mathrm{CI}$ -0.67 to -0.32$), \mathrm{p}<0.01)$. Anxiety was measured with the VAS $(44 \%),{ }^{26} 273236$ STAI $(33 \%),{ }^{313344}$ NRS $(11 \%)^{25}$ or HADS $(11 \%) \cdot{ }^{30}$ We performed a back-transformation on the 11-point VAS/NRS and the STAI scale, since these were most abundantly represented in the pooled data. This yielded a reduction of 1.05 (95\% CI 0.67 to 1.41 ) points on the VAS/NRS for anxiety and 4.00 (95\% CI 2.56 to 5.36) on the STAI. The median duration of the intervention was $30 \mathrm{~min}$ (IQR 20-105). ${ }^{25-2732} 333644$

Four studies offered postoperative music on multiple days. ${ }^{25} 263336$ Pooling of the data collected after the last postoperative music session resulted in a reduction of postoperative anxiety $(\mathrm{SMD}=-0.39(95 \%$ CI -0.64 to
$-0.15), \mathrm{p}<0.01)$. The forest and funnel plots concerned are shown in online supplemental data S4.

\section{Effect of music intervention on postoperative pain}

Pain scores were pooled after the first postoperative music session in six of the 10 studies. $^{26}{ }^{32-34} 36{ }^{39}$ The data from four of these 10 studies could not be pooled due to missing or unreliable means and SDs. ${ }^{28} 293542$ The pooled analysis resulted in a statistically significant effect of perioperatively offered music on postoperative pain ( $\mathrm{SMD}=$ $-0.51(95 \%$ CI -0.84 to -0.19$), \mathrm{p}<0.01)$. Pain was measured with the VAS $(66 \%),{ }^{26} 3233{ }^{36}$ NRS $(17 \%)^{34}$ or VRS $(17 \%) \cdot{ }^{39}$ Back-transformation yielded a reduction of 1.26 (95\% CI 0.47 to 2.07) points on the VAS/NRS for pain. The median duration of the intervention was $30 \mathrm{~min}$ (IQR 28-109).

Three studies offered postoperative music on multiple days. ${ }^{30} 3336$ Pooling of the data collected after the last postoperative music session did not result in a significant effect on pain $(\mathrm{SMD}=-0.40(95 \%$ CI -0.87 to -0.07$)$, 


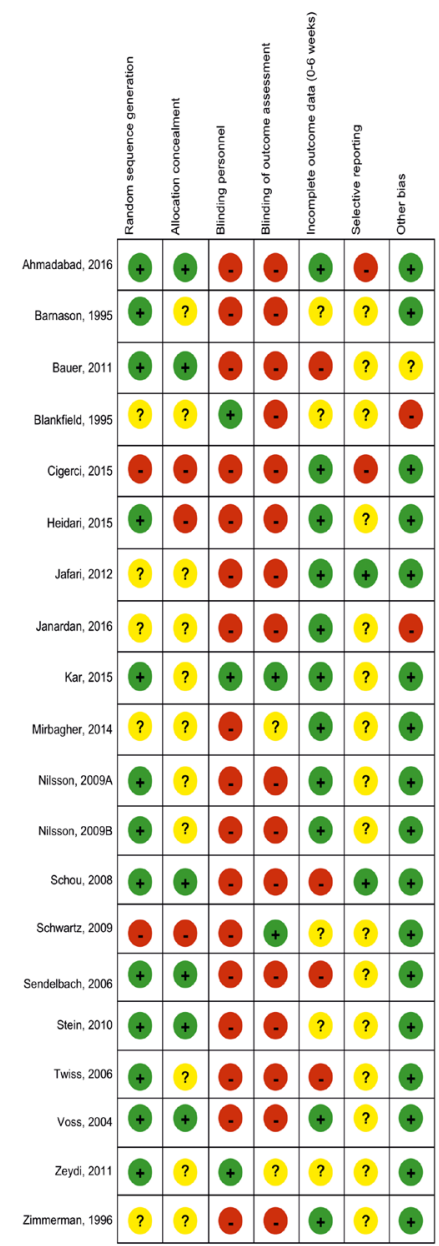

Figure 2 Risk of bias assessment. $\mathrm{p}<0.10)$. The forest and funnel plots concerned are shown in Supplementary data S5).

\section{GRADE rating}

Application of the GRADE criteria led to the following considerations. The risk of bias was moderate to high for both anxiety and pain; the precision was considered low, as effect sizes ranged from low to high within the $95 \%$ CIs of pooled estimates, influencing clinical decision-making. Consistency was considered high for both anxiety and pain, as all studies showed consistent results and all 95\% CIs overlapped. Heterogeneity was statistically significant for pain $\left(\mathrm{I}^{2}=56 \%\right.$ (95\% CI $0 \%$ to $\left.\left.82 \%\right), \mathrm{p}=0.04\right)$ but not for anxiety $\left(\mathrm{I}^{2}=0 \%\right.$ (95\% CI $0 \%$ to $\left.\left.63 \%\right), \mathrm{p}=0.47\right)$; due to the wide CIs, the possibility of substantial heterogeneity cannot be ruled out for either outcome. Directness was considered high, as studies directly targeted the population of interest and reported outcomes critical for decision-making. There was no substantial evidence for publication bias, as scattering in the funnel plots for anxiety and pain was fairly symmetrical. Definite conclusions could not be drawn, however, because the number of studies assessing anxiety and pain was small. In conclusion, the GRADE certainty rating is moderate.

\section{Subgroup analysis}

A combination of preoperative and postoperative music was administered by three studies assessing anxiety ${ }^{30} 3336$ and by two studies assessing pain. ${ }^{336}$ Pooled data analysis of these three and two studies did not result in a statistically significant effect on anxiety $(\mathrm{SMD}=-0.21(95 \% \mathrm{CI}-0.55$ to 0.13$), \mathrm{p}<0.22)$ and pain $(\mathrm{SMD}=-0.57(95 \% \mathrm{CI}-1.45$ to $0.31), p=0.20)$. Five studies assessing anxiety ${ }^{25-27} 3244$ and four studies assessing pain ${ }^{26} 323439$ provided music solely

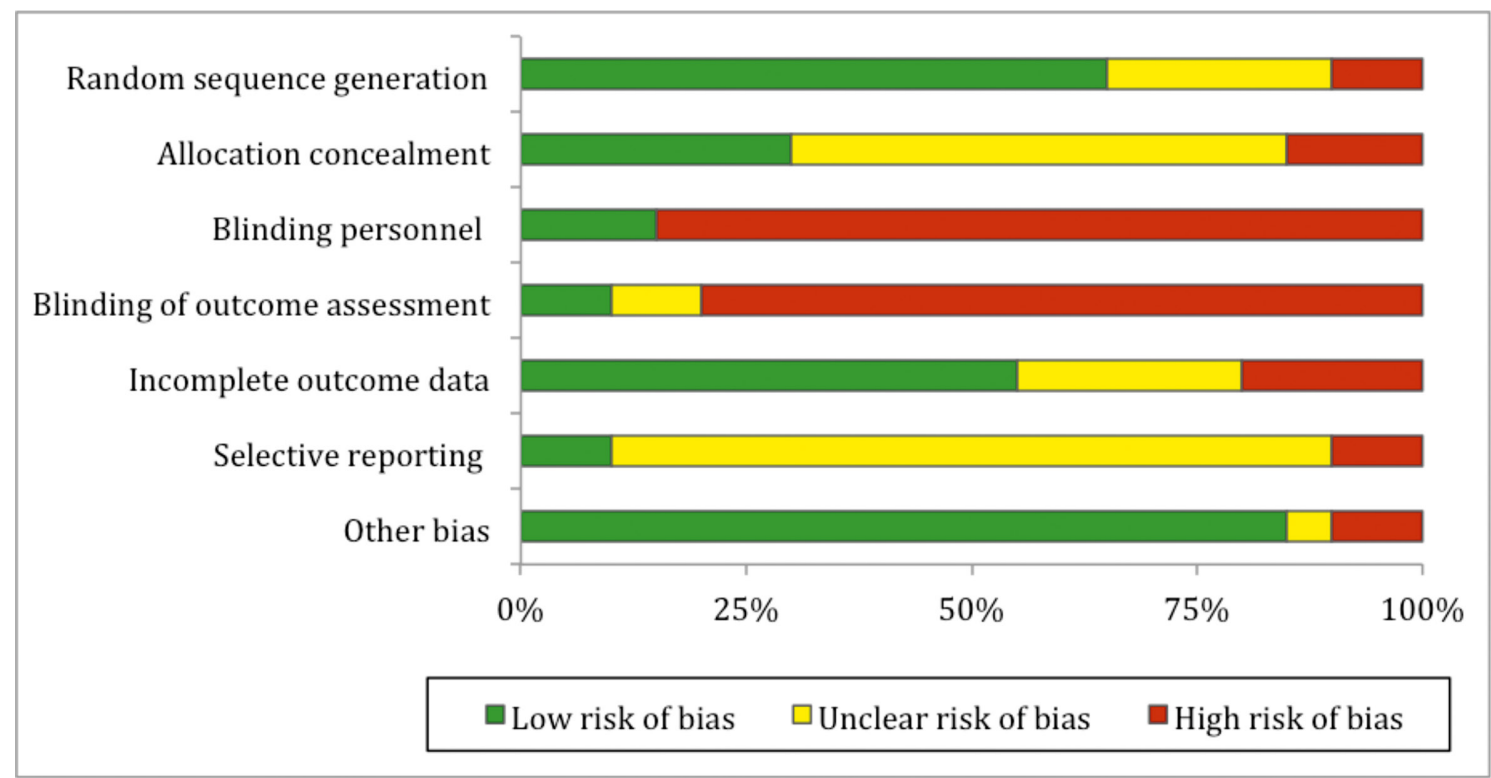

Figure 3 Summary of risk of bias. 
postoperatively. Pooled data analysis of these five and four studies resulted in a statistically significant effect for anxiety $(\mathrm{SMD}=-0.61(95 \% \mathrm{CI}-0.83$ to -0.39$), \mathrm{p}<0.01)$ and pain ( $\mathrm{SMD}=-0.46(95 \% \mathrm{CI}-0.82$ to -0.11$), \mathrm{p}=0.01)$. Two studies assessing anxiety ${ }^{30}{ }^{31}$ provided a combination of intraoperative and postoperative music. Pooled data analysis showed no statistically significant effect on postoperative anxiety (SMD $=-0.30(95 \%$ CI -0.88 to 0.27$)$, $\mathrm{p}=0.30$ ). The information in question was not available for the effect on pain.

Subgroup analysis of the relation between music selection and postoperative anxiety of four studies ${ }^{252632} 36$ in which patients chose from pre-selected music lists resulted in a statistically significant reduction $(\mathrm{SMD}=-0.51(95 \%$ CI -0.77 to -0.25$), p<0.01)$. The same type of analysis of the two studies ${ }^{33} 44$ in which patients provided their own music also resulted in a significant reduction in anxiety $(\mathrm{SMD}=-0.46(95 \% \mathrm{CI}-0.82$ to -0.11$), \mathrm{p}<0.01)$. Pooled data analysis of two studies ${ }^{27}{ }^{30}$ in which music was chosen by the researcher did not show a statistically significant effect on anxiety $(\mathrm{SMD}=-0.41(95 \% \mathrm{CI}-1.26$ to 0.41$)$, $\mathrm{p}=0.32$ ).

Pooled data analysis of five studies ${ }^{26} 32343639$ in which patients chose from provided playlists resulted in a statistically significant reduction in postoperative pain (SMD $=-0.40(95 \%$ CI -0.71 to -0.09$), p=0.01)$. We could not pool data on the effect of patients' self-chosen or researcher-selected music on pain because in only one study could patients select their own music ${ }^{33}$ and in none of the studies did the researcher select the music. Forest plots are shown in online supplemental data S6.

The effect of music on anxiety is statistically significant for both low risk $(\mathrm{SMD}=-0.44(95 \% \mathrm{CI}-0.83$ to -0.05$)$, $\mathrm{p}=0.03)$ and high risk $(\mathrm{SMD}=-0.53(95 \% \mathrm{CI}-0.75$ to $-0.30), \mathrm{p}<0.01$ ) of bias studies based on the randomisation procedure. For pain the same analysis resulted in a non-significant effect for low-risk studies $(\mathrm{SMD}=-0.39$ (95\% CI -0.85 to 0.06$), \mathrm{p}=0.09$ ) and a significant effect for high-risk studies $(\mathrm{SMD}=-0.69(95 \% \mathrm{CI}-1.01$ to $-0.36), \mathrm{p}<0.01)$. Forest plots regarding this are shown in online supplemental data S6.

\section{Effect of music on other parameters}

As written in our PROSPERO protocol, we evaluated the effect of perioperative music interventions on several other parameters. No statistically significant effects were found on perioperative opioid use, length of stay, time on mechanical ventilation, systolic blood pressure, diastolic blood pressure, mean arterial pressure, heart rate, and respiratory rate. Forest plots are shown in online supplemental data S7. The only statistically significant finding was a minimal increase in oxygen saturation in the ICU $(\mathrm{SMD}=0.43$ (95\% CI 0.08 to 0.78$), \mathrm{p}=0.02)$.

\section{DISCUSSION}

It is important to recognise and treat patients' perioperative anxiety, as it has been associated with a higher postoperative pain intensity, lower quality of life, higher benzodiazepine and opioid consumption, and higher morbidity and mortality rates. ${ }^{45-49}$ Regarding the latter, Takagi et al concluded from a meta-analysis that perioperative anxiety correlated with an almost twofold higher late postoperative mortality compared with that in patients without perioperative anxiety. ${ }^{50}$ Also, the use of benzodiazepines for anxiety is controversial. While they are effective in reducing preoperative anxiety and promoting sedation, they have also been associated with a worse postoperative recovery and a higher risk of delirium. ${ }^{51-55}$ Moreover, the use of benzodiazepines and opioids to reduce perioperative anxiety and pain in hospital may lead to chronic use and substance dependency. ${ }^{567}$ Introducing risk-free music interventions for patients undergoing cardiac surgery is most likely cost effective as this may reduce patients' anxiety and pain, and consequently their benzodiazepine and opioid consumption.

This meta-analysis showed a significant beneficial effect of listening to music on postoperative anxiety and pain in patients undergoing cardiac surgery. These results are in line with the previous qualitative review of GraftonClarke $e t a l .{ }^{58}$ We performed the current study in order to systematically review the literature with the help of an information specialist, in order to prevent missing important articles on the subject, and to quantify the cause-effect relationship between music and postoperative anxiety and pain in patients undergoing cardiac surgery and to study the magnitude of the effect of music. Repeated music interventions postoperatively sustain the beneficial effect on anxiety until postoperative day 8 . This effect is also seen when studies were analysed separately based on the risk of bias due to the randomisation procedure, since this was the only risk of bias variable which could be divided into low and high risk of bias. Our review found that exposure to recorded music reduced postoperative anxiety by 1.05 points on the VAS/NRS and 4.00 points on the STAI. A recent RCT comparing the effect of recorded music and that of midazolam on anxiety in patients undergoing a peripheral nerve block found no difference in this respect between the study arms. ${ }^{59}$ Interestingly, midazolam was associated with a reduction of 4.2 points on the STAI, comparable to that which we found for music exposure. In the current study, for pain this reduction was 1.26 points on the VAS/NRS, which was a significant reduction. ${ }^{60}{ }^{61}$ Also, the GRADE certainty was rated moderate in our study, which implies that the authors believe that these estimated clinical relevant effects are probably close to the true effect.

Postoperatively offered music significantly reduced postoperative pain. This effect was not observed when we pooled data of studies providing preoperative music in combination with postoperative music. This was not expected, since preoperative anxiety is associated with postoperative pain. However, the low number of studies administering preoperative music resulted in high variability in the meta-analysis, and potentially led to an underestimation of the effect. Therefore, definite 
conclusions cannot be drawn. Furthermore, this effect was also not observed when studies with low risk of bias and high risk of bias due to the randomisation procedure were analysed separately. Also, music significantly increased the mean oxygen saturation $(\mathrm{SMD}=0.43(95 \%$ CI 0.08 to 0.78 ), $\mathrm{p}=0.02$ ), but this increase was too low to be considered clinically relevant.

In our subgroup analyses on the impact of music selection, the largest beneficial effect on anxiety was seen when patients selected music from preselected lists, followed by self-chosen music. Researcher-selected music had no statistically significant effect on anxiety. Selecting music from a playlist also was associated with a beneficial effect on pain. Only one study assessing pain included self-chosen music, which showed a statistically significant effect when the music was provided in the ICU on postoperative days $1-3 .{ }^{33}$ These findings are mostly in line with those of a meta-analysis of RCTs by Kühlmann $\mathrm{et} \mathrm{al}$, in which selection of music from a preselected list had the largest beneficial effect. ${ }^{15}$ However, they also found a statistically significant effect of researcher-selected music on anxiety and pain. Discrepancies in the magnitude of the music effect between our meta-analysis and that of Kühlmann et al can be explained by the small number of studies included in our subgroup analyses, leading to a potential underestimation of the effect. Therefore, definite conclusions regarding music selection in patients undergoing cardiac surgery cannot be drawn. We did not find the beneficial effect of music on postoperative opioid use described by Fu et al. ${ }^{17}$ This can be explained by the small number of studies assessing opioid usage included in our meta-analysis, and the use of standardised postoperative opioid regimens in these studies.

In more than half of the studies included in our review, music intervention was administered in the ICU. A meta-analysis by Richard-Lalonde et al investigating the effect of music in an adult mixed population admitted to the ICU also resulted in a significant positive effect of music on pain. ${ }^{62}$ More importantly, international guidelines for ICU care recommend offering music to reduce pain and strongly recommend further research of nonpharmacological interventions for the treatment of anxiety and pain, also to prevent delirium. ${ }^{63}$

As mentioned in the results, no effects were found of music on more objective parameters such as opioid use, length of stay, time on mechanical ventilation, systolic blood pressure, diastolic blood pressure, mean arterial pressure, heart rate, respiratory rate, and oxygen saturation. This is probably due to the fact that these parameters were mostly secondary outcome measures and thus no power calculation was performed on these variables. Also, the sample sizes of the studies were relatively low, which results in difficulties drawing definite conclusions.

\section{Strengths and limitations}

A strength of this review is the low heterogeneity between surgical procedures. Furthermore, the VAS and STAI for anxiety and the VAS for pain, which are reliable, validated and easy assessment tools, were the most commonly used tools in the included studies, facilitating the clinical interpretation. The most important limitation was the moderate to high risk of bias. Many studies did not provide sufficient details regarding random sequence generation and allocation concealment. Because our primary outcomes were patient-reported outcomes, the impossibility of blinding of the participants to the music intervention led to a high risk of detection bias. It is only feasible to blind patients when a music intervention is solely administered during general anaesthesia. This was not the case in any of the included studies. Therefore, further research in the cardiac surgical population with music intervention solely being applied during general anaesthesia could have added value. The small numbers of studies included in our subgroup analyses prevented drawing definite conclusions regarding subgroups. Lastly, the timing, duration and type of music intervention varied greatly between studies, and meta-regression analysis could not be performed due to the limited number of studies.

\section{Future research requirements}

Future research on music interventions in cardiac surgery should focus on certain methodological factors. In order to make definite conclusions, multicentre studies with larger sample sizes should be conducted, while most studies included in this meta-analysis have relatively low sample sizes. Furthermore, the risk of selection bias due to sequence generation and allocation concealment is easily solved by using a reliable randomisation method. The risk of performance and detection bias, however, is more difficult to avoid in music intervention studies since awake subjects and personnel are difficult to blind, especially in studies with subjective outcome measures. Lastly, there is still a lot of ambiguity in the current literature on the type of music, duration and frequency of the intervention. Therefore, it would be a great addition if studies with music interventions would report these factors in their studies in order to provide proper guidelines for implementation of music in standard medical care. Until these guidelines are implemented, we recommend use of the published study protocol of the IMPECT trial (Interventions with Music in PECTus excavatum treatment) as a guide for future studies. ${ }^{64}$

\section{CONCLUSION}

This systematic review and meta-analysis of RCTs indicates that perioperatively offered recorded music interventions were associated with a significant reduction in postoperative anxiety and pain in the cardiac surgical population. Music is easily applicable in the perioperative setting and has no known side effects. Therefore, implementation of perioperative recorded music intervention in the standard care of cardiac surgical patients should be considered. 
Acknowledgements The authors thank W Bramer, biomedical information specialist of the Medical Library at the Erasmus University Medical Center, for his assistance in the literature search and Ko Hagoort, Erasmus Medical Center, Rotterdam, for providing editorial advice.

Contributors $\mathrm{JJ}$ conceived the study idea. EK and RJB coordinated the systematic review, screened the abstracts and full texts, wrote the first draft of the manuscript and judged risk of bias in the studies. EK, RJB, JvR, JJMT, MK and JJ interpreted the data and critically revised the manuscript. EK, RJB, JvR, JJMT, MK and JJ had full access to all of the data in the study and can take responsibility for the integrity of the data and the accuracy of the data analysis.

Funding The authors have not declared a specific grant for this research from any funding agency in the public, commercial or not-for-profit sectors.

Competing interests None declared.

Patient consent for publication Not required.

Ethics approval This study does not involve human participants and thus ethical approval was not required.

Provenance and peer review Not commissioned; externally peer reviewed.

Data availability statement Data sharing not applicable as no datasets were generated and/or analysed for this study.

Open access This is an open access article distributed in accordance with the Creative Commons Attribution Non Commercial (CC BY-NC 4.0) license, which permits others to distribute, remix, adapt, build upon this work non-commercially, and license their derivative works on different terms, provided the original work is properly cited, appropriate credit is given, any changes made indicated, and the use is non-commercial. See: http://creativecommons.org/licenses/by-nc/4.0/.

\section{ORCID iDs}

Ellaha Kakar http://orcid.org/0000-0002-7472-308X

Joost van Rosmalen http://orcid.org/0000-0002-9187-244X

\section{REFERENCES}

1 Eisenberg E, Pultorak Y, Pud D, et al. Prevalence and characteristics of post coronary artery bypass graft surgery pain (PCP). Pain 2001;92:11-17.

2 Bjørnnes AK, Rustøen T, Lie I, et al. Pain characteristics and analgesic intake before and following cardiac surgery. Eur $J$ Cardiovasc Nurs 2016;15:47-54.

3 Gan TJ, Habib AS, Miller TE, et al. Incidence, patient satisfaction, and perceptions of post-surgical pain: results from a US national survey. Curr Med Res Opin 2014;30:149-60.

4 De Oliveira GS, Holl JL, McCarthy RJ, et al. Overestimation of mortality risk and preoperative anxiety in patients undergoing elective general surgery procedures: a propensity matched analysis. Int J Surg 2014;12:1473-7.

5 Aslan Özlem, Tosun B. Cardiovascular surgery patients: intensive care experiences and associated factors. Asian Nurs Res 2015;9:336-41.

6 Aslan FE, Badir A, Arli SK, et al. Patients' experience of pain after cardiac surgery. Contemp Nurse 2009;34:48-54.

7 Pavlin DJ, Rapp SE, Polissar NL, et al. Factors affecting discharge time in adult outpatients. Anesth Analg 1998;87:816-26.

8 Ballantyne JC, Carr DB, deFerranti S, et al. The comparative effects of postoperative analgesic therapies on pulmonary outcome: cumulative meta-analyses of randomized, controlled trials. Anesth Analg 1998;86:598-612

9 Vadivelu N, Mitra S, Narayan D. Recent advances in postoperative pain management. Yale J Biol Med 2010;83:11-25.

10 Hirji SA, Landino S, Cote C, et al. Chronic opioid use after coronary bypass surgery. J Card Surg 2019;34:67-73.

11 Head SJ, Milojevic M, Taggart DP, et al. Current practice of state-of-the-art surgical coronary revascularization. Circulation 2017;136:1331-45.

12 Weiss AJ, Elixhauser A. Trends in operating room procedures in US hospitals, 2001-2011. 171. Statistical Brief, 2006.

13 Brandt J, Leong C, Benzodiazepines LC. Benzodiazepines and Zdrugs: an updated review of major adverse outcomes reported on in epidemiologic research. Drugs $R$ D 2017;17:493-507.

14 Weinbroum AA, Szold O, Ogorek D, et al. The midazolam-induced paradox phenomenon is reversible by flumazenil. Epidemiology, patient characteristics and review of the literature. Eur J Anaesthesiol 2001;18:789-97.
15 Kühlmann AYR, de Rooij A, Kroese LF, et al. Meta-analysis evaluating music interventions for anxiety and pain in surgery. $\mathrm{Br} J$ Surg 2018;105:773-83.

16 van der Heijden MJE, Oliai Araghi S, van Dijk M, et al. The effects of perioperative music interventions in pediatric surgery: a systematic review and meta-analysis of randomized controlled trials. PLoS One 2015;10:e0133608.

17 Fu VX, Oomens P, Klimek M, et al. The effect of perioperative music on medication requirement and hospital length of stay: a metaanalysis. Ann Surg 2020;272:961-72.

18 Fu VX, Oomens P, Sneiders D, et al. The effect of perioperative music on the stress response to surgery: a meta-analysis. J Surg Res 2019;244:444-55.

19 Hole J, Hirsch M, Ball E, et al. Music as an aid for postoperative recovery in adults: a systematic review and meta-analysis. The Lancet 2015;386:1659-71.

20 Liberati A, Altman DG, Tetzlaff J, et al. The PRISMA statement for reporting systematic reviews and meta-analyses of studies that evaluate health care interventions: explanation and elaboration. PLoS Med 2009;6:e1000100.

21 Bramer WM, Rethlefsen ML, Mast F, et al. Evaluation of a new method for librarian-mediated literature searches for systematic reviews. Res Syn Meth 2018;9:510-20.

22 Schardt C, Adams MB, Owens T, et al. Utilization of the PICO framework to improve searching PubMed for clinical questions. BMC Med Inform Decis Mak 2007;7:16.

23 Higgins JPT, Thomas J, Chandler J. The Cochrane Handbook for systematic reviews of interventions version 5.1.0, 2011.

24 Guyatt GH, Oxman AD, Vist GE, et al. GRADE: an emerging consensus on rating quality of evidence and strength of recommendations. BMJ 2008;336:924-6.

25 Barnason S, Zimmerman L, Nieveen J. The effects of music interventions on anxiety in the patient after coronary artery bypass grafting. Heart Lung 1995;24:124-32.

26 Bauer BA, Cutshall SA, Anderson PG. Effect of the combination of music and nature sounds on pain and anxiety in cardiac surgical patients: a randomized study. Altern Ther Health Med 2011;17:16-23.

27 Heidari S, Babaii A, Abbasinia M, et al. The effect of music on anxiety and cardiovascular indices in patients undergoing coronary artery bypass graft: a randomized controlled trial. Nurs Midwifery Stud 2015;4:e31157.

28 Nilsson $U$. The effect of music intervention in stress response to cardiac surgery in a randomized clinical trial. Heart Lung 2009;38:201-7.

29 Sendelbach SE, Halm MA, Doran KA, et al. Effects of music therapy on physiological and psychological outcomes for patients undergoing cardiac surgery. J Cardiovasc Nurs 2006;21:194-200.

30 Stein TR, Olivo EL, Grand SH, et al. A pilot study to assess the effects of a guided imagery audiotape intervention on psychological outcomes in patients undergoing coronary artery bypass graft surgery. Holist Nurs Pract 2010;24:213-22.

31 Twiss E, Seaver J, McCaffrey R. The effect of music listening on older adults undergoing cardiovascular surgery. Nurs Crit Care 2006;11:224-31.

32 Voss JA, Good M, Yates B, et al. Sedative music reduces anxiety and pain during chair rest after open-heart surgery. Pain 2004;112:197-203.

33 Ciğerci Y, Ozbayir T. The effects of music therapy on anxiety, pain and the amount of analgesics following coronary artery surgery. Turkish J Thorac Cardiovasc Surg 2016;24:44-50.

34 Jafari H, Emami Zeydi A, Khani S. The effects of listening to preferred music on pain intensity after open heart surgery. Iran $J$ Nurs Midwifery Res 2012;17:1-6.

35 Shorbani S, Azarasa M, Rafiei H. Effect of listening to preferred music on intensity of pain and physiologic parameters in patients undergoing coronary artery bypass grafting surgery. J Nurs Health Sci 2016;5.

36 Schou K. Music therapy for post operative cardiac patients: a randomized controlled trial evaluating guided relaxation with music and music listening on anxiety, pain, and mood. Aalborg: InDiMedia, 2008. forskningsdatabasen.dk

37 Nilsson U. Soothing music can increase oxytocin levels during bed rest after open-heart surgery: a randomised control trial. J Clin Nurs 2009;18:2153-61.

38 Zeydi AE, Jafari $\mathrm{H}$, Khani $\mathrm{S}$. The effect of music on the vital signs and SPO2; of patients after open heart surgery: a randomized clinical trial. J Mazandaran Univ Med Sci 2011;20:72-82.

39 Zimmerman L, Nieveen J, Barnason S, et al. The effects of music interventions on postoperative pain and sleep in coronary artery bypass graft (CABG) patients. Sch Inq Nurs Pract 1996;10:153-70. 
40 Blankfield RP, Zyzanski SJ, Flocke SA, et al. Taped therapeutic suggestions and taped music as adjuncts in the care of coronaryartery-bypass patients. Am J Clin Hypn 1995;37:32-42.

41 Kar SK, Sen C, Goswami A. Effect of Indian classical music (RagA therapy) on fentanyl, vecuronium, propofol requirement and cortisol levels in cardiopulmonary bypass. Br J Anaesth 2012;108:ii216.

42 Mirbagher Ajorpaz N, Mohammadi A, Najaran H, et al. Effect of music on postoperative pain in patients under open heart surgery. Nurs Midwifery Stud 2014;3:e20213.

43 Schwartz FJ. A pilot study of patients in postoperative cardiac surgery. , 2009: 1, 70-4.

44 Janardan $\mathrm{C}$. Influence of music therapy and breathing exercises on anxiety in post-operative cardiac diseased individuals. Int $J$ Physiother 2016;3:22-8.

45 Navarro-Garcia MA, Marin-Fernandez B, Carlos-Alegre de V. Preoperative mood disorders in patients undergoing cardiac surgery: risk factors and postoperative morbidity in the intensive care unit [Trastornos del animo preoperatorios en cirugia cardiaca: factores de riesgo y morbilidad postoperatoria en la unidad de cuidados intensivos]. Rev Esp Cardiol 2011;64:1005-10.

46 Pignay-Demaria V, Lespérance F, Demaria RG, et al. Depression and anxiety and outcomes of coronary artery bypass surgery. Ann Thorac Surg 2003;75:314-21.

47 Tully PJ, Depression BRA. Anxiety, and cardiac morbidity outcomes after coronary artery bypass surgery: a contemporary and practical review. J Geriatr Cardiol 2012;9:197-208.

48 Spence J, Belley-Cote E, Devereaux PJ. Benzodiazepine administration during adult cardiac surgery: a survey of current practice among Canadian anesthesiologists working in academic centres [L'administration de benzodiazepines pendant La Chirurgie cardiaque CheZ I'Adulte: evaluation de la pratique actuelle des anesthesiologistes canadiens exercant en milieu Universitaire]. Can J Anaesth 2018;65:263-71.

49 Hernández-Palazón J, Fuentes-García D, Falcón-Araña L, et al. Assessment of preoperative anxiety in cardiac surgery patients lacking a history of anxiety: contributing factors and postoperative morbidity. J Cardiothorac Vasc Anesth 2018;32:236-44.

50 Takagi H, Ando T, Umemoto T. Perioperative depression or anxiety and postoperative mortality in cardiac surgery: a systematic review and meta-analysis. Heart Vessels 2017;32:1458-68.

51 van Beek S, Kroon J, Rijs K. The effect of midazolam as premedication on the quality of postoperative recovery after laparotomy: a randomized clinical trial [Effet du midazolam en premedication sur la qualité de la recuperation postoperatoire après une laparotomie: une étude clinique randomisée]. Can J Anaesth 2020;67:32-41.

52 Mijderwijk H, van Beek S, Klimek M. Lorazepam does not improve the quality of recovery in day-case surgery patients: a randomised placebo-controlled clinical trial. Eur J Anaesthesiol 2013;30:743-51.

53 Maurice-Szamburski A, Auquier P, Viarre-Oreal V. Effect of sedative premedication on patient experience after general anesthesia: a randomized clinical trial. JAMA 2015;313:916-25.

54 Zaal IJ, Devlin JW, Hazelbag M, et al. Benzodiazepine-associated delirium in critically ill adults. Intensive Care Med 2015;41:2130-7.

55 Kok L, Slooter AJ, Hillegers MH. Benzodiazepine use and neuropsychiatric outcomes in the ICU: a systematic review. Crit Care Med 2018;46:1673-80.

56 Halfens R, Cox K, Kuppen-Van Merwijk A. Effect of the use of sleep medication in Dutch hospitals on the use of sleep medication at home. J Adv Nurs 1994;19:66-70.

57 Brummett CM, Waljee JF, Goesling J, et al. New persistent opioid use after minor and major surgical procedures in US adults. JAMA Surg 2017;152:e170504.

58 Grafton-Clarke C, Grace L, Harky A. Music therapy following cardiac surgery - is it an effective method to reduce pain and anxiety? Interact Cardiovasc Thorac Surg 2019;28:722-7.

59 Graff V, Cai L, Badiola I. Music versus midazolam during preoperative nerve block placements: a prospective randomized controlled study. Reg Anesth Pain Med 2019.

60 Myles PS, Urquhart N. The linearity of the visual analogue scale in patients with severe acute pain. Anaesth Intensive Care 2005;33:54-8.

61 Kelly A-M. The minimum clinically significant difference in visua analogue scale pain score does not differ with severity of pain. Emerg Med J 2001;18:205-7.

62 Richard-Lalonde M, Gélinas C, Boitor M, et al. The effect of music on pain in the adult intensive care unit: a systematic review of randomized controlled trials. J Pain Symptom Manage 2020;59:1304-19.

63 Devlin JW, Skrobik Y, Gélinas C, et al. Clinical practice guidelines for the prevention and management of pain, agitation/sedation, delirium, immobility, and sleep disruption in adult patients in the ICU. Crit Care Med 2018;46:e825-73.

64 Billar RJ, Kühlmann AYR, Schnater JM, et al. Interventions with Music in PECTus excavatum treatment (IMPECT trial): a study protocol for a randomised controlled trial investigating the clinical effects of perioperative music interventions. BMJ Open 2020;10:e036380. 